Contribution à la connaissance

\title{
du cycle de Lepidauchen stenostoma Nicoll, 1913 (Trematoda, Digenea, Lepocreadiidae Nicoll, 1935, Lepocreadiinae Odhner, 1905)
}

\author{
par Georges PREVOT
}

(Laboratoire de Zoologie Générale, Faculté des Sciences de Marseille)

\section{Résumé}

Les deux Gastéropodes Prosobranches Nassa incrassata et Pisania maculosa hébergent la métacercaire, libre, de Lepidauchen stenostoma. La description de cette dernière est donnée de façon détaillée. L'adulte a été trouvé en Méditerranée chez deux Labridés : Julis giofredi et Crenilabrus pavo. Sa description est complétée par l'observation d'une vésicule séminale externe et d'un réceptacle séminal. Elle est attribuée à la super-famille des Lepocreadioidea, famille des Lepocreadiidae, sous-famille des Lepocreadiinae.

\section{Summary}

Contribution to the life history of Lepidauchen stenostoma (Trematoda, Digenea, Lepocreadiidae Nicoll, 1935, Lepocreadiinae Odhner, 1905).

The two prosobranch gastropods Nassa incrassata and Pisania maculosa harbour the metacercaria, not encysted, of Lepidauchen stenostoma. Its detailed description is presented. Adult has been found from two Labridae: Julis giofredi and Crenilabrus pavo. Its description is perfected by adjunction of an external seminal vesicle and a seminal receptacle. That species is attributed to the Lepocreadioidea, Lepocreadiidae, Lepocreadiinae. 
Mes recherches sur les Trématodes larvaires parasites de Prosobranches marins m'ont conduit à la découverte chez ces derniers d'une métacercaire particulièrement intéressante par l'évolution avancée de son appareil génital. J'ai pu l'attribuer au genre Lepidauchen, attribution confirmée par la présence de l'adulte chez des Poissons marins.

Le genre Lepidauchen a été créé par Nicoll (1913) à partir de deux spécimens adultes découverts dans l'intestin de Labrus bergylta Ascanius de la région de Plymouth (Manche). Nicoll leur a donné le nom de Lepidauchen stenostoma. Cette espèce n'a d'ailleurs, à ma connaissance, jamais plus été signalée depuis.

\section{METACERCAIRE}

Les hôtes de cette larve sont deux Gastéropodes: Pisania maculosa Lamarck et Nassa incrassata Müller. Seules les Pisania récoltées à l'entrée du Vieux-Port de Marseille étaient parasitées. L'examen de nombreux spécimens provenant de diverses localités avoisinantes s'est toujours montré négatif. Sur un total de 157 Pisania examinées, 7 seulement étaient parasitées $(4,4 \%)$, en général par une métacercaire, parfois par deux.

Cette dernière n'est pas enkystée. Elle se localise dans ia masse viscérale du Mollusque, généralement au niveau du tube digestif, et n'est entourée d'aucune réaction mélanique.

Les Nasses parasitées proviennent de draguages effectués au large de Marseille sur un fond coralligène. L'examen de Nasses récoltées en divers lieux de la région (en particulier les ports) s'est toujours montré infructueux.

Sur un total de 18 Nassa incrassata, 3 étaient parasitées (16,5\%), l'une d'entre elles par deux métacercaires, les deux autres par une seule. Leur localisation est tout à fait identique à celle décrite chez Pisania maculosa.

\section{Description.}

MATÉRIEL DE DESCRIPTION :

10 métacercaires dont 7 de Pisania et 3 de Nassa. Je n'ai pas noté de différences de taille ou de maturation selon l'origine de ces larves.

Elles ont une couleur jaunâtre et sont animées de mouvements très faibles aussi bien à l'intérieur de l'hôte que dans un liquide physiologique.

CoRps (fig. 1) :

Leur grande taille $(940-1.600 \times 500-870) *$ permet de les découvrir aisément chez le Mollusque. La cuticule est pourvue d'épines serrées dans la région antérieure. Leur densité diminue vers l'arrière, elles disparaissent totalement au niveau de la ventouse ventrale.

* N.B. - Toutes les mesures sont données en microns. 


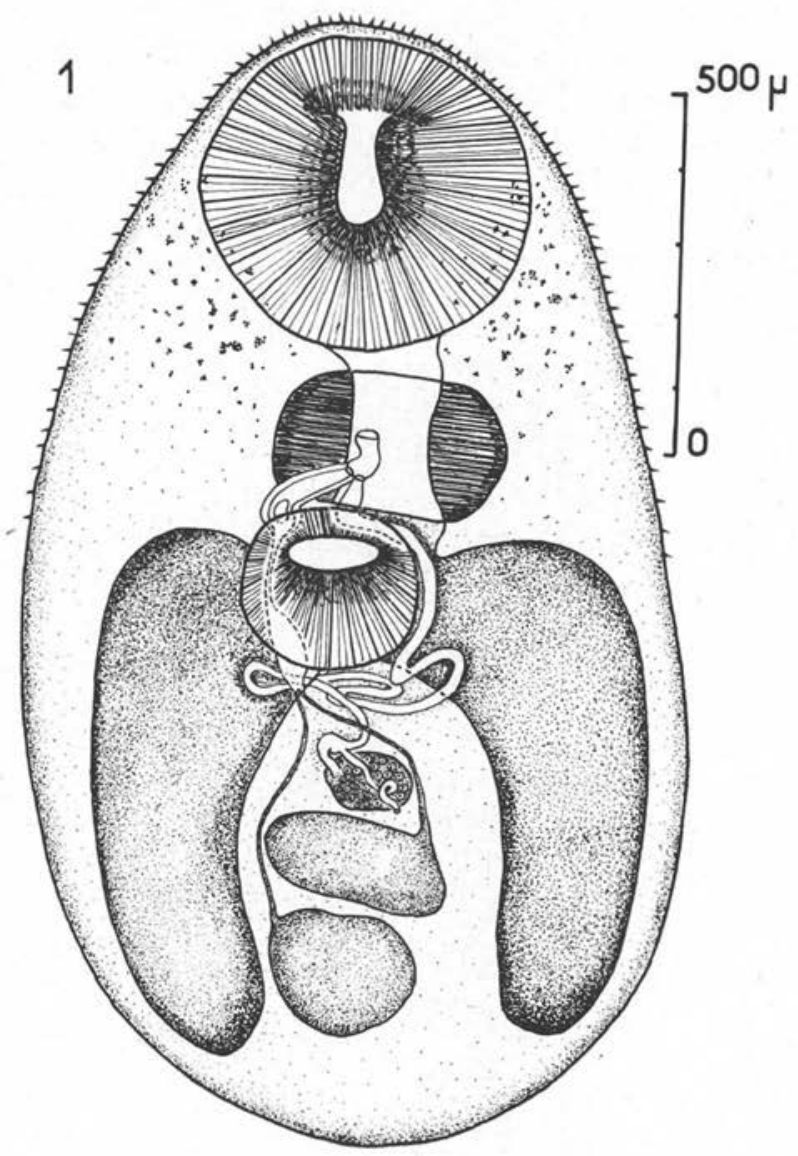

Fig. 1. - Organisation générale de la métacercaire, vue ventrale

\section{TACHES OCULAIRES :}

Elles sont visibles sous la forme d'une poussière de petits grains disposés en face dorsale, latéralement à la ventouse orale et au pharynx. Les métacercaires jeunes présentent des taches oculaires moins fragmentées et plus visibles que les formes plus âgées.

\section{Ventouses :}

La ventouse orale est sub-circulaire, un peu plus allongée transversalement. Elle mesure $240-400 \times 270-450$. La ventouse ventrale nettement plus petite est circulaire : 160 à 240 de diamètre. Elle se situe à mi-distance entre les deux extrémités. Le rapport ventousaire varie peu. Il augmente légèrement avec la taille du ver : 1,60-1,85. 
ApPaReIL Digestif :

L'ouverture buccale a une forme très caractéristique. C'est une fente longitudinale s'ouvrant sur la face ventrale au centre de la ventouse orale. Le prépharynx est très court : 30 à 60 . Le pharynx volumineux, musculeux, est plus large que haut : 135-180 $\times 200-285$.

Esophage absent. Les caecums digestifs, latéraux, très larges, sont sub-terminaux. Leur diamètre diminue progressivement vers l'arrière depuis l'acétabulum.

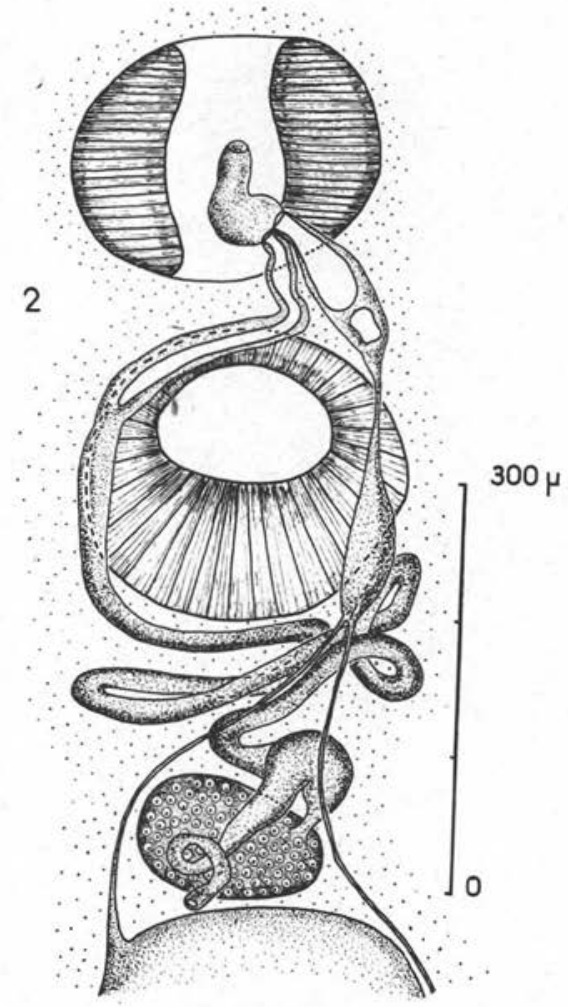

Fig. 2. - Détail de l'appareil génital de la métacercaire, vue dorsale

ApPaReil génital (fig. 2) :

Appareil génital mâle :

Les deux testicules généralement ovoïdes sont allongés transversalement. Ils sont disposés en tandem entre les deux caecums. Souvent contigus, ils peuvent cependant être légèrement séparés ou se chevaucher sur une petite partie.

Le testicule postérieur occupe la région située un peu en avant de la terminaison 
des caecums $(90-180 \times 110-260)$. Le testicule antérieur se situe au milieu de la zone post-acétabulaire $(90-165 \times 130-285)$. Les spermiductes se rejoignent en arrière de la ventouse ventrale au niveau du renflement d'une vésicule séminale externe légèrement déjetée sur la droite. Un fin canal la met en communication avec la poche du cirre disposée au niveau du bord antérieur droit de l'acétabulum. Cette dernière est partagée en deux parties, l'une postérieure, correspondant à la vésicule séminale interne, la seconde antérieure, au pénis.

\section{Appareil génital femelle:}

L'ovaire, de forme ovoïde, est situé en avant du testicule antérieur dans une position médiane. Comme j'ai pu l'observer pour les testicules, sa taille varie avec le degré de maturité de la larve : 40-75 $\times 90-150$. Son grand axe est transversal. En avant et sur la droite se détache un court oviducte issu de sa face dorsale. Ce dernier, cilié sur toute sa longueur, débouche dans une portion élargie correspondant à un petit réceptacle séminal. Le canal de Laurer, de grande taille, s'ouvre dorsalement au niveau de l'ovaire. L'ootype n'est pas visible. L'utérus décrit plusieurs boucles, toutes comprises entre l'ovaire et la ventouse ventrale. Il se dirige ensuite vers l'avant, passant sur le bord de l'acétabulum, pour se terminer au niveau du pharynx par un métraterme.

\section{Atrium génital :}

Ses parois sont très fines, sa taille petite. Il s'ouvre sur la face ventrale par un pore génital médian situé à la limite de la seconde moitié du pharynx.

APPAReil eXCrÉteur (fig. 3):

La vessie excrétrice de grande taille comprend une partie impaire remontant jusqu'à l'ovaire et s'ouvrant à l'extérieur par un pore urinaire précédé d'un sphincter. Deux branches de diamètre décroissant remontent latéralement jusqu'au niveau du bord antérieur du pharynx. De chacune de ces branches se détachent deux canaux aboutissant à de nombreux groupes de protonéphridies.

Je n'ai pu déterminer la formule excrétrice n'ayant pas suffisamment de matériel, cependant j'ai pu observer dans la région postérieure les protonéphridies groupées par ensembles de quatre. Le nombre de ces dernières, très élevé, permet d'envisager une formule excrétrice d'un type assez complexe.

\section{ADULTE}

Mes recherches sur la faune intestinale des Poissons fréquentant la zone où ont été trouvées les Nasses parasitées m'ont permis de rencontrer l'adulte de cette métacercaire de Lepidauchen chez deux espèces de Labridés: Julis giofredi Risso et Crenilabrus pavo Brünn*.

* Plusieurs exemplaires provenant du rectum de Coris julis m'ont été aimablement transmis par M. Oliver du C.S.U. de Perpignan. Les Poissons ont été pêchés sur un fond coralligène. Il apparaît bién que cette espèce de Lepidauchen est assez rare, mais possède une vaste répartition géographique.

Annales de Parasitologie humaine et comparée (Paris), t. 43, 1968, $\mathrm{n}^{\circ} 3$. 
Sur 93 Julis giofredi disséquées, 4 seulement étaient parasitées (4,3\%), tandis que sur les 2 Crenilabrus pavo, 1 était parasité. La dissection de 11 C. ocellatus et de 2 C. grisaeus s'est montrée négative. Trois des quatre Girelles renfermaient un seul parasite, la quatrième en renfermait deux. Le Crénilabre hébergeait deux Trématodes dans son tube digestif. Il n'y a pas de localisation particulière dans l'intestin de l'hôte.

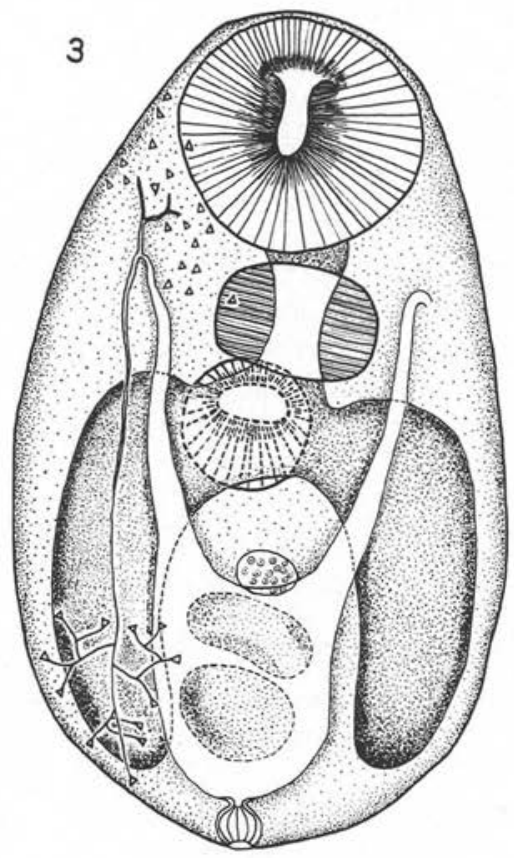

Fig. 3. - Appareil excréteur

Le petit nombre de métacercaires trouvées ne m'a pas permis de réaliser l'infestation expérimentale de l'un de ces hôtes définitifs. Il est intéressant de constater que le tube digestif des Girelles et des Crénilabres disséqués renfermait fréquemment des débris de coquilles appartenant à Nassa incrassata.

Je dois signaler que sur les sept exemplaires trouvés, un seul avait atteint sa maturité complète. Les six autres se trouvaient à un degré d'évolution plus ou moins avancé.

\section{Description.}

Ces Trématodes présentent des mouvements de peu d'importance. Ils adhèrent faiblement aux villosités intestinales desquelles on peut les détacher aisément.

Leur morphologie (fig. 4) diffère assez peu de celle de la métacercaire, exception faite pour les organes génitaux. 


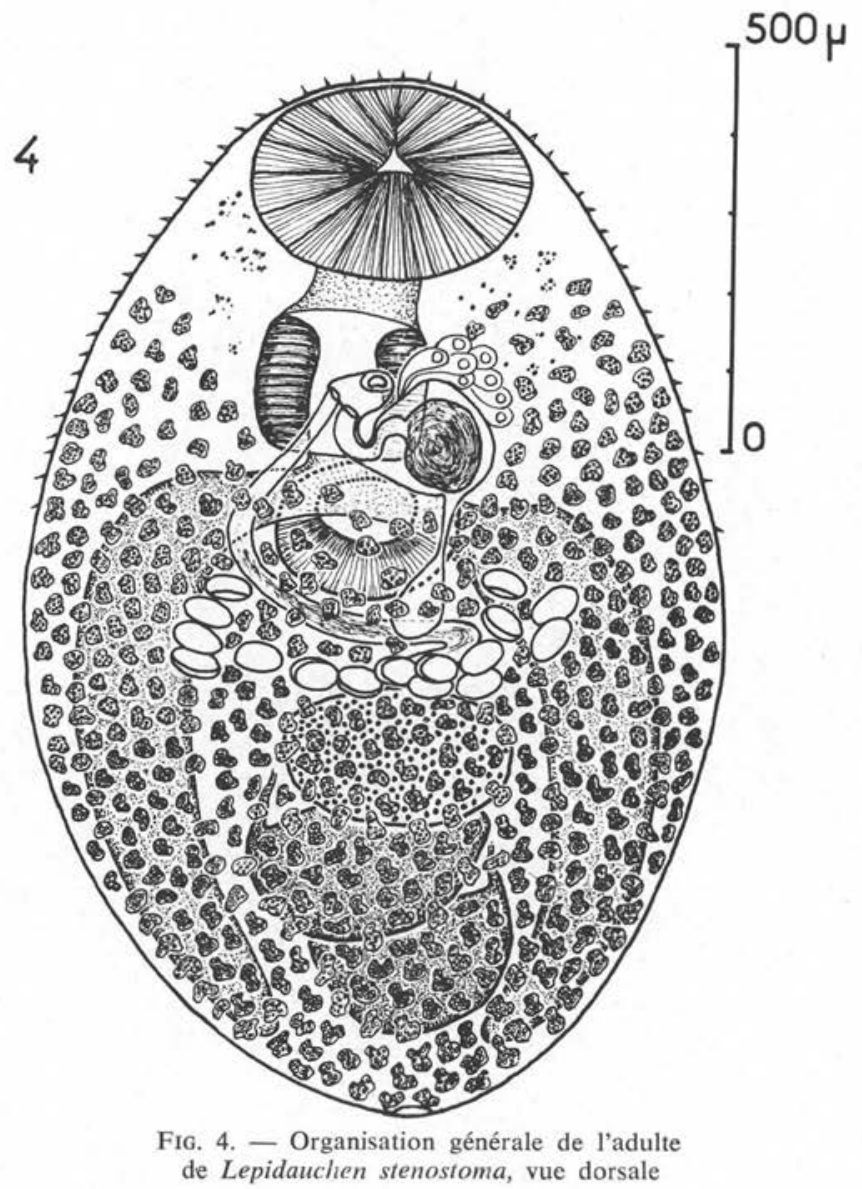

La taille est sensiblement identique à celle des grosses larves $(1.150-1.500 \times 600$ 850). Il n'y a pas de croissance allométrique.

Les taches oculaires sont encore visibles sur les parties latérales de la ventouse orale. Cette dernière, plus large que haute, mesure $270-360 \times 290-400$; la ventouse ventrale 160 à 190 de diamètre. Le rapport ventousaire oscille entre 1,60 et 1,90.

L'appareil digestif n'offre rien de remarquable par rapport à celui de la larve, sinon qu'il est généralement coloré par des débris alimentaires provenant du Poisson. Appareil GÉNItal (fig. 5) :

\section{Mâle :}

La taille des testicules s'est légèrement accrue. Ils mesurent 120-165 $\times$ 210-310 pour le testicule antérieur et $120-180 \times 185-265$ pour le testicule postérieur. Ils sont 
disposés en tandem, le testicule antérieur recouvrant légèrement le postérieur. Les canaux déférents se rejoignent au niveau d'une vésicule séminale externe à paroi fine, remplie de spermatozoïdes. Cette dernière communique avec la poche du cirre par un canal étroit de longueur variable suivant l'état de contraction du ver. La poche du

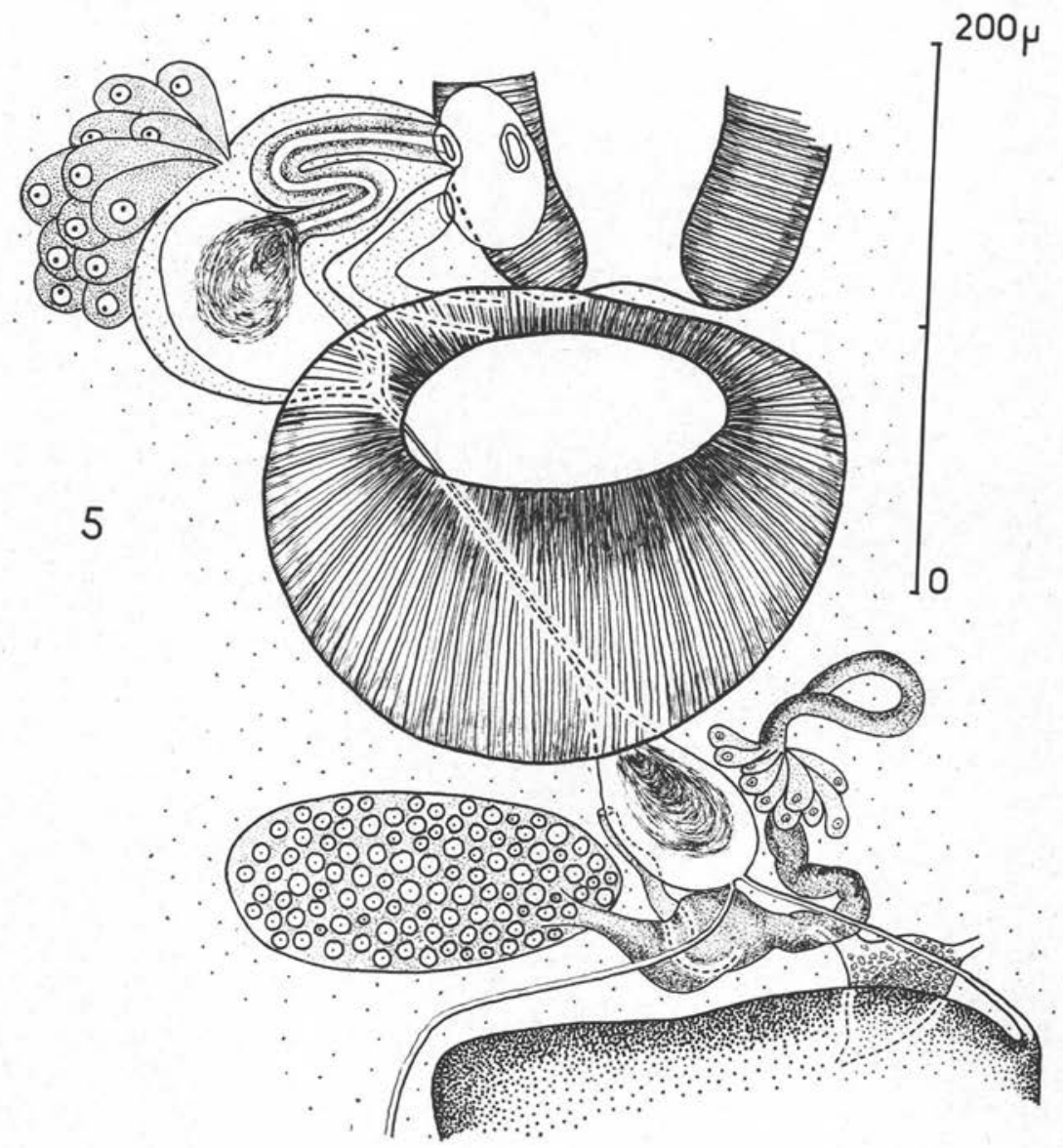

Fig. 5. - Détail de l'appareil génital de l'adulte, vue ventrale

cirre est bien développée, de forme un peu arrondie ; elle mesure environ 135 de large pour 225 de long. On distingue à l'intérieur une vésicule séminale interne, sphérique, précédant le pénis replié sur lui-même. Au niveau de la jonction entre la vésicule séminale et le cirre débouchent des glandes prostatiques de très grande taille situées à l'extérieur de la poche du cirre. Je n'ai pas observé de glandes prostatiques au niveau de la vésicule séminale externe. 


\section{Femelle :}

L'ovaire s'est développé considérablement par rapport à celui de la larve. Sa taille varie entre $80-129 \times 153-270$. Il se détache de sa face dorsale un court oviducte aboutissant à un petit réceptacle séminal dépourvu de spermatozoïdes. Dans ce dernier aboutit le canal de Laurer s'ouvrant sur la face dorsale au niveau de l'ovaire. L'utérus reçoit le vitelloducte impair. Les follicules vitellins s'étendent sur toute la région post-acétabulaire jusqu'à la partie terminale du corps. Ils atteignent dans la région médiane le bord antérieur de la ventouse ventrale et remontent latéralement jusqu'à la limite antérieure du pharynx.

L'ootype est bien visible. Les boucles utérines sont toutes situées entre l'ovaire et l'acétabulum. Les deux dernières, remplies de spermatozoïdes, forment un réceptacle séminal utérin. Elles se terminent par un métraterme à paroi légèrement épaissie. Les œufs, très peu nombreux, sont operculés et pourvus d'un petit mucron sur le pôle opposé. Ils mesurent: $72-78 \times 40-46$.

\section{Atrium génital :}

Il se situe sur la face ventrale, en-dessous du pharynx. Sa petite taille et la faible épaisseur de ses parois rendent son observation difficile. Le pore génital, médio-ventral, s'ouvre à la limite antérieure de la deuxième moitié du pharynx.

\section{DISCUSSION}

La description de ce Trématode est en accord avec la diagnose du genre Lepidauchen donnée par Yamaguti (1958). Je soulignerai cependant certaines différences. Ce sont :

- la présence chez l'espèce étudiée d'un petit réceptacle séminal,

- d'une vésicule séminale externe,

- de cellules prostatiques disposées autour de la poche du cirre.

Deux espèces de Lepidauchen sont actuellement connues:

- L. stenostoma Nicoll, 1913, de Labrus bergylta Ascanius, décrite à partir de deux spécimens trouvés dans la Manche (Plymouth);

- L. hysterospina Manter, 1931, de Lagodon rhomboüdes Linnaeus de la côte Atlantique des Etats-Unis (Beaufort, Caroline du Nord). Ce dernier entre en synonymie avec Distomum sp. Linton, 1905 du même hôte à Beaufort.

L. hysterospina peut être éliminé en raison de sa spinulation moins développée antérieurement et présente à l'extrémité postérieure. De plus, il possède une poche du cirre plus allongée et des vitellogènes ne remontant pas au-delà de l'extrémité postérieure du pharynx.

L'espèce étudiée, malgré sa taille moitié moindre, se rapproche de L. stenostoma. Bien que Nicoll ne mentionne pas dans sa description l'existence de la vésicule séminale externe ni du réceptacle séminal, j'assimile cette espèce à Lepidauchen stenostoma Nicoll, 1913. 
Il convient de remarquer que Manter n'indique pas, dans la description de son espèce, la présence d'une vésicule séminale externe. Il n'est pas exclu que cette dernière soit passée inaperçue en raison de la finesse de ses parois et de son intrication avec le réceptacle séminal utérin.

La position systématique du genre Lepidauchen est très discutée par les auteurs :

- Ben Dawes (1947) le place dans la famille des Allocreadiidae Stossich, 1904, et dans la sous-famille des Lepocreadiinae Odhner, 1905.

- Caballero y Caballero (1952) dans les Acanthocolpidae Lühe, 1909, Acanthocolpinae Lühe, 1906.

- Yamaguti le fait entrer dans les Allocreadiidae, sous-famille des Allocreadiinae Looss, 1902.

L'existence chez Lepidauchen d'une métacercaire non enkystée à cuticule spinulée, des taches oculaires et de la vessie excrétrice à paroi fine, me conduit à placer ce genre dans la super-famille des Lepocreadioidea Cable, 1956. Celle-ci est caractérisée par des cercaires ophtalmotrichocerques non enkystées, se développant dans des rédies chez des Gastéropodes Prosobranches. La paroi de leur vessie excrétrice est très fine avec de petites granulations agglomérées en masses. La cuticule est spinulée au moins chez les adultes.

Dans la super-famille des Lepocreadioidea, Skrjabin et Koval distinguent sept familles dont l'une, les Lepocreadiidae Nicoll, 1935, offre les caractères présentés par Lepidauchen. Ce sont :

- les circonvolutions utérines disposées entre l'ovaire ou le testicule antérieur et la ventouse ventrale,

- Ia présence d'une poche du cirre,

- les branches intestinales se terminant en cul-de-sac sans former d'ouverture anale,

- le corps de forme ovale allongée,

- les testicules disposés en tandem ou en diagonale.

Parmi les dix sous-familles définissant les Lepocreadiidae, c'est aux Lepocreadiinae Odhner, 1905, que se rattache le genre Lepidauchen.

Cette sous-famille se caractérise par:

- un pore génital médian s'ouvrant en avant de la ventouse ventrale,

- une poche du cirre avec vésicule séminale,

- une vésicule séminale externe,

- des branches intestinales se terminant en cul-de-sac,

- des circonvolutions utérines localisées entre l'ovaire et la ventouse ventrale.

Le genre Lepidauchen s'ajoutera donc aux cinq genres définissant selon Skrjabin et Koval les Lepocreadiinae. Ce sont: Eocreadium Szidat, 1954, Lepocreadioides Yamaguti, 1936, Opechonoïes Yamaguti, 1940, Opechona Looss, 1907, et Lepocreadium Stossich, 1904. 
La connaissance du cycle vital complet de ce Trématode définirait évidemment beaucoup mieux sa position systématique, c'est pourquoi je me propose d'orienter mes recherches vers les Gastéropodes Prosobranches en vue de découvrir son premier hôte intermédiaire.

\section{Bibliographie}

DAwes (B.), 1947. - The Trematoda of British Fishes. London printed for the Ray Society, 364 pages.

Caballero y Caballero (E.), 1952. - Revision de los generos y especies que integran la Familia Acanthocolpidae Lühe, 1909 (Trematoda : Digenea). Rev. Med. Veter. Parasit., Tomo $\mathrm{XI}, \mathrm{n}^{\circ} 1-2,231$ pages.

CABLE (R. M.), 1956. - Opistholebes diodontis n. sp., its development in the final host, the affinities of some Amphistomatous Trematodes from the marine fishes and the Allocreadioid problem. Parasit., 46, 1-2, p. 1-13.

-, 1963. - Marine cercariae from Curaçao and Jamaïca. Z. Parasitkde, 23, 429-469.

Dollfus (R. Ph.), 1949. - Sur une cercaire Ophtalmoxiphidiocerque Cercaria isopori et sur la délimitation des Allocreadioidea. Ann. Parasit. Hum. et Comp., 24, 5-6, p. 424435.

Linton (E.), 1905. - Parasites of fishes of Beaufort, North Carolina. Bull. U.S. Bur. Fish (1904), 24, p. 321-428.

Manter (H. W.), 1931. - Some Digenetic Trematodes of marine fishes of Beaufort, North Carolina. Parasit., 23, 3, p. 396-411.

Nicoll (W.), 1913. - New trematode parasites from fishes of the English Channel. Parasit., $5,238-246$.

Sertner (P. G.), 1951. - The life history of Allocreadium ictaluri, Pearse, 1924 (Trematoda : Digenea). J. Parasit., 37, 3, p. 223-244.

SKRJABIN (K. I.) et al., 1964. - Keys to the trematodes of animals and man. University of Illinois Press, 351 pages.

YAMAGUTI (S.), 1958. - Systema Helminthum. Vol. 1. The digenetic trematodes of vertebrates. Part 1 and 11, 1.575 pages, New-York, London. 